\title{
On Separation of Charges and Formation of Linear Structures in the Nuclei of Dislocations in Metals
}

\author{
V. L. Busov \\ Donbass State Engineering Academy, Kramatorsk, Ukraine \\ Email: vlbusov49@gmail.com
}

How to cite this paper: Busov, V.L. (2020) On Separation of Charges and Formation of Linear Structures in the Nuclei of Dislocations in Metals. Applied Mathematics, 11, 739-752.

https://doi.org/10.4236/am.2020.118049

Received: July 9, 2020

Accepted: August 15, 2020

Published: August 18, 2020

Copyright ( 2020 by author(s) and Scientific Research Publishing Inc. This work is licensed under the Creative Commons Attribution International License (CC BY 4.0).

http://creativecommons.org/licenses/by/4.0/ (c) (i) Open Access

\begin{abstract}
In metals, the non-adiabatic mechanism of charge separation into the electron, cation, and vacancy chains is considered. It includes: 1) the photoelectric effect caused by bremsstrahlung caused by the scattering of conduction electrons by impurity ions lying at the ends of these chains and oscillating with the frequency of the local phonon mode; 2) knocking out from the metal cations of the matrix, sufficiently slow (in comparison with the conduction electrons) photoelectrons that pull these cations from the nodes, thereby not violating the electroneutrality condition in the model of a free electron gas as with the static state of the chains and at their motion (slipping, recrawling) in the absence of a thermal field; 3 ) induced radiation along the line of chains; 4) the formation of linear structures in the spatial lattice of octahedral interstitial sites. The conditions for the formation of quasistable bound states in the dislocation core are considered. A hypothesis is advanced that the latent energy in the dislocation cores contains a component that is of an electromagnetic nature.
\end{abstract}

\section{Keywords}

Nonadiabatic Process, Bremsstrahlung, Photoelectric Effect, Photoelectrons, Induced Radiation, Quasistable Bound States

\section{Introduction}

It is known that in metals near edge dislocations there is a difference in bulk density above and below the slip plane [1] [2] [3]. For the ground state of a free electron gas in metals [4], the Fermi energy $\varepsilon_{F} \sim n_{e}^{2 / 3}$, where $n_{e}$ is the bulk concentration of conduction electrons, is the same at all points of the crystal. 
Near the dislocation in the compacted sections above the slip plane, there is a lack of electrons, and in the rarefaction region there is an excess, which leads to a violation of electroneutrality and the appearance of an electric dipole with a dipole moment distributed along the dislocation line and directed along the normal to the slip plane. On the other hand, the solution of the problem of scattering of conduction electrons near the line of edge [5] and screw [6] dislocations is well known, where it was assumed that, firstly, the scattering of conduction electrons by cations is elastic due to its large mass in comparison with the mass of the electron and, accordingly, the negligible recoil momentum and displacement of the cation at the lattice node during scattering, the energy of an electron in a collision can be assumed to be unchanged; secondly, the solution was obtained at large distances from the dislocation line $\rho \approx 2 a_{0} \div 25 a_{0}$ ( $a_{0}$ is the lattice parameter) with the help of asymptotic wave functions and Friedel basis functions [7].

Here it is shown that, first of all, regardless of the type of dislocation around its line, an oscillating distribution of the excess electric charge of the conduction electrons arises $q_{s c r}, q_{e d g} \sim r^{-5 / 2}$ ( $r$ is the distance from the dislocation line to the collision point) in difference from scattering by a charged impurity ion: $q_{\text {imp }} \sim r^{-3}$; secondly, for an edge dislocation, $q_{e d g} \sim \sin \beta \cdot \sin \left(2 k_{F}+\frac{\pi}{4}\right)$, where $k_{F}$ is the modulus of the Fermi wave vector, $\beta$ is the angle between the Burgers vector and the radius vector $\boldsymbol{r}$, which corresponds to the deformation field of the defect $r^{-1}$; for a screw dislocation $q_{s c r} \sim r^{-5 / 2} \cos \left(2 k_{F}+\Phi\right)$, where $\Phi$ is the phase shift, which reflects the axial symmetry of the defect. However, the distribution $q_{s c r}, q_{e d g}(r)$ [5] [6] caused by the deformation field of the defect $r^{-1}$ in the region of a good crystal does not allow us to reliably determine $q_{s c r}, q_{e d g}(r)$ inside the cylindrical region around the defect line with the radius $r \approx 0.1 \div 0.2 \mathrm{~nm}$, as well as the shape, height and width of the potential barrier in these nuclei. In addition, as noted by Friedel [7], the conduction electrons are fast particles and, when passing near the dislocation, they do not settle on the cations of the dislocation core, but only change the direction of their motion, creating a dynamic picture of charge redistribution. As an attempt to remedy the situation, work [8] is known, where in the tight-binding approximation, the solution of the Schrödinger time equation was found by numerical methods for a two-dimensional distorted lattice of cross section perpendicular to the dislocation line considered in the Peierls-Nabarro model. Here, the distribution of the local density of states $N_{0}(\varepsilon)$ of electrons on the energy scale $\varepsilon$ is obtained, which contains two pronounced high peaks, one of which is located in the region $\varepsilon<0$ and the other at $\varepsilon>0$, while the point $\varepsilon=0$ divides the intermediate segment between the peaks, for example, for the s-s bond and the various spatial dependences of the transfer integrals $T(R), \boldsymbol{R}=\boldsymbol{\rho}-\boldsymbol{\rho}^{\prime}$ in the nearest-neighbor approximation: for the power $T \sim R^{-5}$ in the relation 1 (in $\varepsilon>0$ ) to 1.5 (in $\varepsilon<0$ ); at an exponential $T \sim \exp (-\alpha R)-0.5 / 1.5$. In the same time there is no interpretation of this form of $N_{0}(\varepsilon)$. The results of the numerical 
calculation show the decisive role of the dislocation core for the electronic structure of the defect in comparison with the effect of the long-range part of the deformation potential [5] [6].

It is also known ([9], p. 99) that, in crystals of the $\mathrm{NaCl}$ type, edge dislocations in the $\{111\}$ plane with a Burgers vector $\frac{a_{0}}{2}\langle 110\rangle$ and a linear (running) charge density $Q_{\mathrm{NaCl}}= \pm e / 2 a_{0}$ are charged. In semiconductor crystals, particularly in n-type germanium and silicon, a chain of unpaired valence electrons along the dislocation line forms a negative charge density $Q_{s c}, Q_{m}<Q_{s c}<Q_{\mathrm{NaCl}}$ ([9], p. 101). In metals, according to [10] [11], this density is $Q_{m} \approx-(0.01 \div 0.1) e / a_{0}$ in contrast to [5] [6], where the total charge of excess density $q_{\text {scr }}, q_{\text {edg }}$ in volume around the dislocation line at $V_{d} \rightarrow \infty$ tends to zero.

The purpose of this paper is to identify the mechanism for separating the charges of electrons and metal cations, which allows the generation of dislocation nuclei, and the formation of linear structures, as well as the determination of the conditions for the appearance of quasistable bound states in the dislocation core.

\section{Theoretical Model}

First we note that the electric charge, as well as energy, momentum, etc. is the integral of motion ([12], p. 505) is preserved in space and in time. Generation of dislocations occurs mainly in a perfect crystal, where the total charge $\sum_{i} q_{i}$ in its individual volumes and in the entire volume is initially zero. Therefore, after generating the defect $\sum_{i} q_{i}=0$. In addition, as a result of the movement (slipping, recrawling), a single defect leaves no trace in the crystal except for extrusions and intrusions on its free surface. If we consider the separation of charges as a short-lived state for a characteristic period of time $T$ within the trial volume $V$, then the condition $\sum_{i} q_{i}=0$ must be softened, averaging it according to

$$
\left\langle\sum_{i} q_{i}\right\rangle_{V, T}=0 .
$$

On the other hand, the separation of charges in metals mainly occurs under the influence of an external electromagnetic field, penetration of which into the material is possible only within the skin layer. The question arises: "How does the charge separation occur when generating dislocation nuclei outside the skin layer?" Here it should be noted that practically all dislocation models, known in the literature, reflect adiabatic processes, i.e. at the beginning metal cations are shifted, and then electrons are "adjusted" for these displacements. In this paper, we abandon this approach and state hypothesis 1: The processes of generation and motion of the cationic chain, as well as of the entire core of the dislocation as a whole, are nonadiabatic. Here, we will use an intermittent field of electromagnetic nature as the generating field of defect nuclei. At the first stage, we shall single out the main scheme of the charge separation mechanism during generation, where as an intermittent field we take the bremsstrahlung of the 
conduction electrons, which form the halo of the charge $q_{s c r}, q_{\text {edg }}$, when a charged impurity ion moves along a certain periodic law. Here, for simplicity, we use the harmonic oscillations $\cos \Omega t \quad(\Omega$ is the frequency of the natural oscillations of the impurity ion as a local phonon mode ([13], p. 358). This radiation can cause a photoelectric effect for electrons of internal L, K-shells, thus forming a sufficiently slow (in comparison with the conduction electrons) photoelectrons, as well as induced radiation along the dislocation line and long-wavelength secondary photons that go to the region of a good crystal where they are elastically scattered on the metal cations. In other words, we arrive at the problem of scattering of bremsstrahlung photons on bound electrons L, K-shells, and in the absence of secondary photons to resonant scattering ([14], p. 384).

To determine the initial generation conditions, let us use the experiment [15] to determine the electromagnetic emission (EME) in the process of intermittent deformation in an AlMg6Mn aluminum alloy (Al-6.03 wt\% Mg-0.5\% Mn), where the samples were stretched at a constant rate of increase of the applied stress $\sigma$. This alloy is a solid solution of $\mathrm{Mg}$ and $\mathrm{Mn}$ in an aluminum matrix, and in the first stage the influence of $\mathrm{Mn}$ is neglected. The light impurity atom $\mathrm{Mg}\left(M_{\mathrm{Mg}}<M_{\mathrm{Al}}\right)$ gives both valence electrons to the subsystem of the collectivizated conduction electrons of the whole alloy and forms an single impurity ion $\mathrm{Mg}^{2+}$ [16], which creates a local oscillation mode with a frequency [13]

$$
\omega_{L}=\omega_{\max } \sqrt{1-\epsilon^{2}}
$$

at $M_{\mathrm{Mg}}=M_{\mathrm{Al}}(1-\epsilon)$ and $\epsilon>0$ without taking into account the change in the force constant. We note that the axis along which the impurity ion oscillates has a stereographic projection equally distributed in a full solid angle and has a spherical polarization as $t \rightarrow \infty$. Under the actual conditions of the alloy, the homogeneous distribution of the cations $\mathrm{Mg}^{2+}$ takes place on average, for which the average distance $R_{\mathrm{Mg}}$ between them is much larger than the distance $r_{l m}$ of the amplitude of the oscillations decrease to zero: $R_{\mathrm{Mg}} \gg r_{l m}$. If a weak impact (shock) is applied to the free surface of the alloy sample, the displacement of the ion $\mathrm{Mg}^{2+}$ is different from the displacements of the surrounding cations $\mathrm{Al}^{3+}$ at $t=0$ due to the difference in ionic radii $r_{\text {ion }}\left(\mathrm{Al}^{3+}\right)=0.057 \mathrm{~nm}$ and $r_{\text {ion }}\left(\mathrm{Mg}^{2+}\right)=0.074 \mathrm{~nm}$ without taking into account the influence of the coordinate number ([17], p.75), which leads to the replacement of the spherical polarization of the oscillations by linear polarization. In addition, the main feature of [15] for $\sigma=\dot{\sigma}_{0} \cdot t, \dot{\sigma}_{0}=$ const is the linear increase in the EME pulse amplitude $\varphi_{\mathrm{EME}}(t)$ in time $t$, and the time intervals between neighboring pulses, especially in its central part are practically equidistant. We recall that the energy stored in the crystal as a result of pumping at time $t$ is numerically determined by the area under the $\sigma-\varepsilon$ curve, which allows us to replace the time coordinate of the energy coordinate: $\varphi_{\text {EME }}(\varepsilon)$.

\subsection{Bremsstrahlung}

Let us consider the law of conservation of energy and momentum ([14], p. 387) 
in the scattering of conduction electron by an impurity ion

$$
p_{1 \mu}-p_{2 \mu}=k_{\mu}-q_{\mu}(\mu=0,1,2,3),
$$

where $p_{1 \mu}, p_{2 \mu}, k_{\mu}$ and $q_{\mu}$ are the 4-momentum of the incident electron, the scattered electron, the bremsstrahlung photon and the recoil of the ion nucleus, respectively. For the time components of these pulses, expression (3) is written in the form

$$
\varepsilon_{1}-\varepsilon_{2}=\varepsilon_{\gamma}+\varepsilon_{n r}
$$

$\varepsilon_{1}, \varepsilon_{2}, \varepsilon_{\gamma}$ and $\varepsilon_{n r}$ are the kinetic energy of the initial and final states of the electron, the photon energy of bremsstrahlung and the recoil of the nucleus of the impurity ion, respectively. For the spatial components of 4-momenta

$$
\boldsymbol{p}_{1}-\boldsymbol{p}_{2}=\boldsymbol{k}-\boldsymbol{q}
$$

In the limiting case of low energies of primary electrons from $\varepsilon_{F}=3-10 \mathrm{eV}$ in metals up to tens of $\mathrm{keV}$ in the problem of the continuous spectrum of $\mathrm{x}$-rays, the photon momentum $|\boldsymbol{k}|$ small compared with the momentum of the electron in view of the practically zero rest mass of the photon, and there fore

$$
\boldsymbol{q}^{2}=\left(\boldsymbol{p}_{1}-\boldsymbol{p}_{2}\right)^{2}
$$

In the nonrelativistic case, with the initial velocity of the electron $\left|v_{e}\right| \ll c, c$ is the speed of light, the differential bremsstrahlung cross section is equal to the product of the Rutherford elastic scattering cross section $\mathrm{d} \sigma_{s}$ by the photon emission probability $\mathrm{d} w_{\gamma}$

$$
\mathrm{d} \sigma_{\gamma}=\mathrm{d} \sigma_{s} \cdot \mathrm{d} w_{\gamma}
$$

where

$$
\mathrm{d} w_{\gamma}=\frac{\alpha}{(2 \pi)^{2}}\left[\frac{\boldsymbol{k}}{\varepsilon_{\gamma}}\left(\boldsymbol{v}_{e 1}-\boldsymbol{v}_{e 2}\right)\right]^{2} \frac{\mathrm{d} \varepsilon_{\gamma}}{\varepsilon_{\gamma}} \mathrm{d} o,
$$

and $\alpha$ is the fine structure constant, $\alpha \approx 1 / 137 ; \boldsymbol{v}_{e 1}$ and $\boldsymbol{v}_{e 2}$ are the velocity vectors of the primary and scattered electron; do is the solid angle element. Here, the radiation intensity $I$ reaches a maximum in the direction perpendicular to the plane of motion of the electron $\left(\boldsymbol{p}_{1}, \boldsymbol{p}_{2}\right)$ i.e. when $\boldsymbol{k}$ lies in this plane. In accordance with the classical theory of radiation, $\mathrm{d} w_{\gamma}$ is the ratio of the intensity of the dipole radiation $I_{d}$ at low frequencies to the photon energy $\varepsilon_{\gamma}$ equal to the average number of photons $n_{\gamma}$ per unit time. We note that the representation of the bremsstrahlung cross section in the form (7), (8) is possible in all those cases in which low-frequency photons are emitted. In this connection, the theoretical spectrum of bremsstrahlung in a metal, for example, in aluminum ([14], p. 398), is of interest in the form of the dependence $I_{d}$ on $\varepsilon_{\gamma} / T_{e} \quad\left(T_{e}\right.$ is the kinetic energy of the primary electron), taking into account the electron shielding of the inner shells of the impurity ion, which allows one to eliminate the divergence as $\varepsilon_{\gamma} \rightarrow 0$ in the process of determining the total cross section $\sigma_{\gamma}$ with the help of (7), (8). Here, in the interval $\varepsilon_{\gamma}=(0.2 \div 0.8) T_{e}$, the value 
of $I_{d}$ (in relative units) varies from 7 to 4 , i.e. in engineering calculations is replaced by an average of 5.5, and for light elements of type $\mathrm{Al}$ screening is of little significance. It becomes clear from the expressions (3)-(8) that by changing the components of the 4-momentum of the impurity nucleus $q_{\mu}$ in time by an external action, leading to jumps $\left[q_{0}\right]$ and $[\boldsymbol{q}]$, wherein it is possible to achieve level jumps $\left[I_{d}\right]$, where the jump $[\boldsymbol{q}]$ creates a discontinuous displacement of the ion nucleus [l] along an arbitrary axis $l$. To do this, we place single impurity ion in an ideal crystal at a distance $R$ from its free surface, with $R \gg a_{0}$. We divide the entire space of the crystal into two half-spaces by a plane parallel to its free surface. Here we note that according to the theory of periodic chains of bonds between atoms and estimates of the surface energy of the free surface of the crystal ([18], p. 14), the crystallographic orientation of the crystal must correspond to the most densely packed crystallographic plane. In our case of metals with a face-centered lattice this is a family of slip planes $\{111\}$ in Al. Here, the light ion $\mathrm{Mg}^{2+}$ is in an octahedral interstitial site with six nearest neighbors-cations $\mathrm{Al}^{3+}$ ([3], p. 122). The total recoil momentum $\sum_{j} \boldsymbol{q}_{j}$ that imparts to the impurity nucleus the conduction electrons scattered on it from the lower half-space $\sum_{j} \boldsymbol{q}_{j}$ (LHS) is equal in magnitude and opposite in sign to the analogous momentum transferred from electrons from the upper half-space $\sum_{j} \boldsymbol{q}_{j}$ (UHS) without taking into account the influence of thermal oscillations and in the absence of external forces. As already noted, there exists a local mode of oscillations of the ion $\mathrm{Mg}^{2+}$ with a frequency $\omega_{L}$ by gap from the upper spectral phonon band. Here, the dipole moment of the charge system "impurity ion-distribution of scattered electrons" is equal to zero due to the symmetry of the lattice, while the stereographic projection of the ion oscillation axis is uniformly distributed in a full solid angle in time $t \gg T_{L}=2 \pi / \omega_{L}$. If a weak impact (shock) is made on the free surface of the crystal along the normal to it, then the recoil momentum $\boldsymbol{q}_{n r}$ given to the nucleus of the single impurity is equal to the difference $\sum_{j} \boldsymbol{q}_{j}$ (LHS) (the coincidence of the directions of the external force action and the recoil of the ion nucleus from scattering electrons) and $\sum_{j} \boldsymbol{q}_{j}$ (UHS) (the above directions are opposite)

$$
\boldsymbol{q}_{n r} \equiv[\boldsymbol{q}]=\sum_{j} \boldsymbol{q}_{j}(\mathrm{LHS})-\sum_{j} \boldsymbol{q}_{j}(\mathrm{UHS})
$$

and arises in the form of a jump $[\boldsymbol{q}]$. Here it should be noted that under the influence of the impact both the impurity ion and its nearest and subsequent cations $\mathrm{Al}^{3+}$ are displaced, where for the easy $\mathrm{Mg}^{2+}$ this displacement is obviously larger. As a result, such a impact (shock) lowers the point symmetry of the lattice to the axial symmetry near the impurity ion, but all the characteristics of a free electron gas under translational invariance must correspond to periodic boundary conditions within a three-dimensional cube with the side $L \gg a_{0}$, and the time interval $\tau_{e} \approx L / v_{F} \quad\left(v_{F}\right.$ is Fermi velocity) within the framework of this model is proportional to the relaxation time of the free-electron subsystem and plays the role of the time for its "adjustment" to the lattice change for a free path length of order $L$ and collisions with one impurity ion. On the other hand, the 
time interval for the amplitude shift of the impurity ion $\Delta t_{\mathrm{Mg}} \approx a_{0} / v_{s} \quad\left(v_{s}\right.$ is the sound velocity) for a perfect aluminum single crystal at $L \approx 10^{-2} \mathrm{~m}$ and $v_{F} \gg v_{s}$, and for the intervals $\tau_{e}$ and $\Delta t_{\mathrm{Mg}}$, the condition $\Delta t_{\mathrm{Mg}} \ll \tau_{e}$ is satisfied. In this case, the charged ion is displaced with respect to the spherically symmetric distribution of the excess density $q_{\text {edg }}, q_{\text {scr }}$ of the charge of the conduction electrons, and a dipole moment of the system arises at the time $t=\Delta t_{\mathrm{Mg}}$

$$
P_{s}=\left(k_{m}-k_{i m p}\right) \cdot e \cdot \Delta z_{\mathrm{Mg}-\mathrm{Al}},
$$

where $k_{m}$ and $k_{i m p}$ are valences of matrix and impurity atoms, respectively; $\Delta z_{\mathrm{Mg}-\mathrm{Al}}$ is the displacement amplitude of the Mg ion with respect to the nearest neighbors, $\Delta z_{\mathrm{Mg}-\mathrm{Al}} \approx(0.01 \div 0.1) \cdot a_{0}$. Hence, the bremsstrahlung intensity $I_{d}$ in the dipole approximation ([19], p. 206) for an single impurity is

$$
I_{d}=\frac{e^{2}\left(k_{m}-k_{i m p}\right)^{2}}{3 c^{3}} \cdot \omega_{L}^{4} \cdot\left(\Delta z_{\mathrm{Mg}-\mathrm{Al}}\right)^{2} .
$$

In a real aluminum polycrystalline with a grain dispersion of $10 \div 100 \mu \mathrm{m}$, the values of $\tau_{e}$ and $\Delta t_{\mathrm{Mg}}$ are of the same order $\Delta t_{\mathrm{Mg}} \cong \tau_{e}$, but $\Delta t_{\mathrm{Mg}}>\tau_{e}$. Here, in the expression (11), the frequency $\omega_{L}$ should be replaced by the plasma frequency $\omega_{p}$ with implicit replacement of the mass $M_{\text {ion }}$ by $m_{e}$, where the intensity $I_{d}$ increases by $5-6$ orders of magnitude and the excitation of cation energy levels and knock out photoelectrons becomes possible.

\subsection{Photoelectric Effect}

Let us consider the scheme of the energy levels of the $\mathrm{Mg}$ atom ([12], p. 558), analogous to the scheme of the $\mathrm{Al}$ atom. Here we can distinguish two concepts: the binding energy $\varepsilon_{i}$ and the ionization energy $I_{i}$. For an single atom $\varepsilon_{i}$ coincides in absolute value with the length of a energy segment from zero to a level corresponding to a given $s, p, d, \ldots$ electron in the region $\varepsilon<0$, and the quantity $I_{i}>\varepsilon_{i}$ in the region $\varepsilon>0$ corresponds to the electron state as $r \rightarrow \infty, r$ is the distance from the nucleus. In the crystal, the definition of $\varepsilon_{i}$ remains unchanged, and the determination of $I_{i}$ as a function of $r$ essentially depends on the degree of screening by the conduction electrons. In dielectrics and semiconductors, the Coulomb interaction of the knocked out electron and the resulting cation can be neglected even at $10 a_{0}<r<100 a_{0}$. In metals, this interval is $5 a_{0}<r<10 a_{0}$. Consider the absorption of the bremsstrahlung photon $\gamma_{b r}$ by the electron of the internal L, K-shells of the cation $\mathrm{Al}^{3+}$ in the case when the photon energy $\varepsilon_{\gamma}$ exceeds $\varepsilon_{i}$ and the knocked out electron passes to the continuous spectrum region for $\varepsilon>0$, i.e. there is a photoelectric effect. In this case, the photoelectron in the nucleus of the dislocation must reach a region roughly $a_{0}<r<2 a_{0}$.

The most accurate description of the photoelectric effect is given by quantum electrodynamics ([14], p. 360), where in the nonrelativistic case an angular dependence of the differential photoelectric cross section for unpolarized incident photons $\gamma_{b r}$ 


$$
\mathrm{d} \sigma_{\text {phef }} \sim \sin ^{2} \vartheta,
$$

where $\vartheta$ is the angle between the polarization vectors of the photon $\boldsymbol{e}_{\gamma}$ and the photoelectron momentum $\boldsymbol{p}_{\text {phe }}$. For polarized incident photons $\gamma_{b r}$ is replaced by

$$
\sin ^{2} \vartheta \rightarrow \cos ^{2} \vartheta=\sin ^{2} \vartheta \cdot \cos ^{2} \varphi
$$

where $\varphi$ is the angle between the planes $\left(\boldsymbol{p}_{\text {phe }}, \boldsymbol{k}_{\gamma}\right)$ and $\left(\boldsymbol{k}_{\gamma}, \boldsymbol{e}_{\gamma}\right)\left(\boldsymbol{k}_{\gamma}\right.$ is the wave vector of the incident photon $\gamma_{b r}$. Analysis (12), (13) shows that the majority of photoelectrons emerge in the direction of polarization vector $\boldsymbol{e}_{\gamma}$ of the incident photon $\gamma_{b r}$ (condition*). The total cross section of the photoelectric effect on the K-shell in the Born approximation ([14], p. 362) has the form

$$
\sigma_{K}=A_{K} \cdot \varepsilon_{\gamma}^{-7 / 2},
$$

where $A_{K}=A_{0} \cdot I_{i 1}^{5 / 2}(\mathrm{Al}) ; A_{0}=64 \alpha / 3 m_{e} ; I_{i 1}(\mathrm{Al})$ is the ionization energy of the $\mathrm{Al}$ atom on the K-shell. In the photoelectric effect on the L-shell, the total cross section breaks up into a cross section when it is absorbed by two 2s-electrons

$$
\sigma_{L_{I}}=A_{1} \cdot \varepsilon_{\gamma}^{-4}+A_{2} \cdot \varepsilon_{\gamma}^{-5},
$$

and the cross section for absorption by six $2 \mathrm{p}$-electrons

$$
\sigma_{L_{I I}}+\sigma_{L_{I I I}}=A_{3} \cdot \varepsilon_{\gamma}^{-5}+A_{4} \cdot \varepsilon_{\gamma}^{-5},
$$

where $A_{1}\left(I_{i 2}^{m_{1}}\right), A_{2}\left(I_{i 2}^{m_{2}}\right), A_{3}\left(I_{i 2}^{m_{3}}\right), A_{4}\left(I_{i 2}^{m_{4}}\right)$ have the same structure as $A_{K}$, but different numerical coefficients and exponents $m_{j}$ for $I_{i 2}(\mathrm{Al})$-the ionization energy of the $\mathrm{Al}$ atom on the $\mathrm{L}$-shell, and $I_{i 2}(\mathrm{Al})<I_{i 1}(\mathrm{Al})$. Here it should be noted that when $\varepsilon_{\gamma}-\varepsilon_{i} \rightarrow 0$, when the kinetic energy of photoelectrons is small in comparison with $\varepsilon_{\gamma}, \varepsilon_{i}$, resonance scattering takes place ([14], p. 384), where a narrow band is cut out from the continuous bremsstrahlung spectrum, which when knocking out photoelectrons from the internal L, K-shells of the cation $\mathrm{Al}^{3+}$ corresponds to the line absorption spectrum. This is indicated by a comparison of the power dependences of the bremsstrahlung cross sections $\sigma_{b r}$ and $\sigma_{K}, \sigma_{L_{n}}$ on $\varepsilon_{\gamma}$ in (8) and (14)-(16).

\subsection{Induced Radiation}

According to the definition, the frequency, phase, polarization and direction of propagation of the electromagnetic wave incident on the metal cation coincide with the same characteristics as the absorbed cation, and after its excitation of the emitted wave. Hence, the energy of the induced radiation wave will be transferred from one cation $\mathrm{Al}^{3+}$ to the neighboring cation without losses, until the wave reaches an impurity ion $\mathrm{Mg}^{2+}$, in which another scheme of energy levels, which leads to elastic scattering back. If the normals of the effective scattering cross sections of two impurity ions lie within the same line, then a standing wave of stimulated or induced radiation arises. We assume that parallel to the free surface of aluminum is a slip plane, where at the nodes of the square grid of oc- 
tahedral interstices there are impurity ions and standing waves of induced radiation appear in each section of grid cells under the influence of a weak shock and bremsstrahlung.

\subsection{Separation of Charges and Bound States in the Dislocation Core}

Let us consider the crystallography of one of the slip planes (111) of a face-centered lattice, which intersects the lines of the main diagonals of the cells of this lattice along the $\langle 111\rangle$ directions normal to this plane within one cell. If the bremsstrahlung photon $\gamma_{b r}$ is transverse: its wave vector $\boldsymbol{k}_{\text {phe }} \perp \boldsymbol{n}_{(111)}$ ( $\boldsymbol{n}_{(111)}$ is the unit vector of the normal to the (111) plane) lies in this plane, and its the polarization vector $\boldsymbol{e}_{b r}$ is directed along [111] $\| \boldsymbol{n}_{(111)}$, then the photon $\gamma_{b r}$ can produce a photoelectric effect on the inner shells of the first cation $\mathrm{Al}^{3+}$, which intersects $\boldsymbol{e}_{b r}$ on the main diagonal of the cell. The photoelectron $e_{p h e}$ ejected by the photon $\gamma_{b r}$ moves according to the condition $\left(^{*}\right)$ in the direction $\boldsymbol{e}_{b r}$ and depending on the level of the kinetic energy $T_{p h e}$ obtained from the photon, it can settle on the first counter and on the subsequent interstitial sites. The possible states of photoelectrons are due to the joint influence of the Coulomb attraction of the cation from which they were knocked out, and the spherically symmetric potential of attraction of the octahedral interstice as a force center. To describe these states and the possible structure arising under the influence of bremsstrahlung, we use the definition of stable bound and quasistable bound states ([19], p. 671), where, for the particle system, in our case, the systems of metal cations and photoelectrons, first, their relative the motion is finite for a long time in comparison with the periods typical for the given system; second, for the formation of bound states, it is necessary to have attractive forces, which for cations and photoelectrons is natural. If the range of distances on which these particles are attracted is separated by an energy potential barrier from the region in which they repel, then the particles can form stable states. Such states lie in the energy region $\varepsilon<0$, for $\varepsilon>0$ they do not exist. However, in the region $U_{0}<\varepsilon<U_{b}$ ( $U_{0}$ is the depth of the potential well, $U_{b}$ is the height of the potential barrier), for some values of $\varepsilon$ there can exist quasi-stable bound states, the lifetime of $\tau_{q w s b} \sim w_{t t}^{-1}$ which is determined by the probability of the tunnelling transition $w_{t t}$ through potential barrier. In this connection, we can disclose the physical essence of the local density of electron states $N_{0}(\varepsilon)$ [8], where a quasi-periodic sequence of shallow minima occurs between a high lower peak at $\varepsilon<0$ and a high upper peak at $\varepsilon>0$ for a power-law dependence of the transport integral. With the help of this sequence and the whole of $N_{0}(\varepsilon)$, we can qualitatively estimate the shape of the potential relief on the energy scale in the dislocation core if we symmetrically reflect the upper half-plane of the graph $N_{0}(\varepsilon)$ on its lower half-plane with respect to the energy axis or, more simply, turn the sheet with the graph by $180^{\circ}$. Such modeling of the potential relief shows that the lower peak corresponds to a narrow potential well of a rectangular shape with a depth of $U_{01}$, and the upper one cor- 
responds to the same well with a depth of $U_{02}$, and $U_{02}>U_{01}$. Inside the intermediate interval between the peaks, firstly, there is a weak growth of the regular component of the potential relief towards upper peak, and secondly, at the beginning and at the end of this energy interval, the Fourier components of the above sequence, oscillating against the background of the regular component, are substantially higher than analogous components within the interval. The linear structure along the $\langle 111\rangle$ and $\boldsymbol{e}_{b r}$ directions, which does not contradict the potential relief of the dislocation core obtained from the numerical calculation [8], is shown in Figure 1. Here at position $A$ there is a site vacancy, at position $B$ in octahedral interstice-cation $\mathrm{Al}^{3+}$, which passed from node $A$, to position near the node $C$-photoelectron, at position $D$-the nearest cation $\mathrm{Al}^{3+}$, displaced under the total influence of vacancy $A$, cation $B$ and photoelectron $C$. Note that the distance between the positions $A$ and $B$ is $b_{0}=\sqrt{2} \cdot a_{0} / 2$, between $B$ and $C$ does not exceed $\leq b_{0}$, and the distance $C D$ is determined by the interval $r_{\text {ion }}\left(\mathrm{Al}^{3+}\right)<R<b_{0}$; all of the matrix metal cations following position $D$ shift from the position $C$ to the depth of the material in accordance with the law $r^{-1}$.

Under the influence of the bremsstrahlung causing the photoelectric effect, the subsequent formation of two systems of bound charges in the space lattice of the octahedral interstices is taking place., where the first system consists of a chain of photoelectrons near the nodes $C$ with a linear charge density $Q_{\text {phe }}=-k_{\text {phe }} \cdot e / a_{0} \quad$ ( $k_{\text {phe }}$ is the number of knocked out photoelectrons, $\left.k_{\text {phe }}=1,2,3\right)$ and chains of metal matrix cations in interstices $B c$ linear density after the departure of photoelectrons from the inner shells of these cations $Q_{w e}=+k_{\text {phe }} \cdot e / a_{0}$. The second system in the model of a free electron gas (jelly model) contains an inhomogeneity in the form of a "ridge" of bulk density $\delta n$ with a linear density $Q_{\delta n}=+3 e / a_{0}$ in the interstices $B$, and along the nodes $A$ with $Q_{v c h}=-3 e / a_{0}$ i.e. "valley" in the homogeneous distribution of a positive charge smeared in space, whose density is equal in magnitude and opposite in sign to the mean charge density of conduction electrons, so that the system of a free electron gas is always electrically neutral. Hence the total linear charge density of the cationic chain $Q_{c c h}=Q_{w e}+Q_{\delta n}$.

To determine the mutual arrangement of the above chains in the face-centered lattice $\mathrm{Al}$, we use the crystallography of the $\{111\}$ slip plane and the system of the main diagonals $\{111\}$ ([20], c. 24). We assume that translational invariance is preserved along the dislocation line between the impurity ions bordering it. Hence, along the dislocation line, a periodic sequence of the above linear structures arises, both along the normal to the chosen plane (111), and in the plane itself along the normal to the dislocation line.

The analysis shows that consideration of the Coulomb effect of cationic and vacancy chains with distributed linear charges on the photoelectron chain in the defect nucleus should be carried out together with the influence of the distributed excess charge $q_{\text {scr }}, q_{e d g}$ of conduction electrons. The model representation 


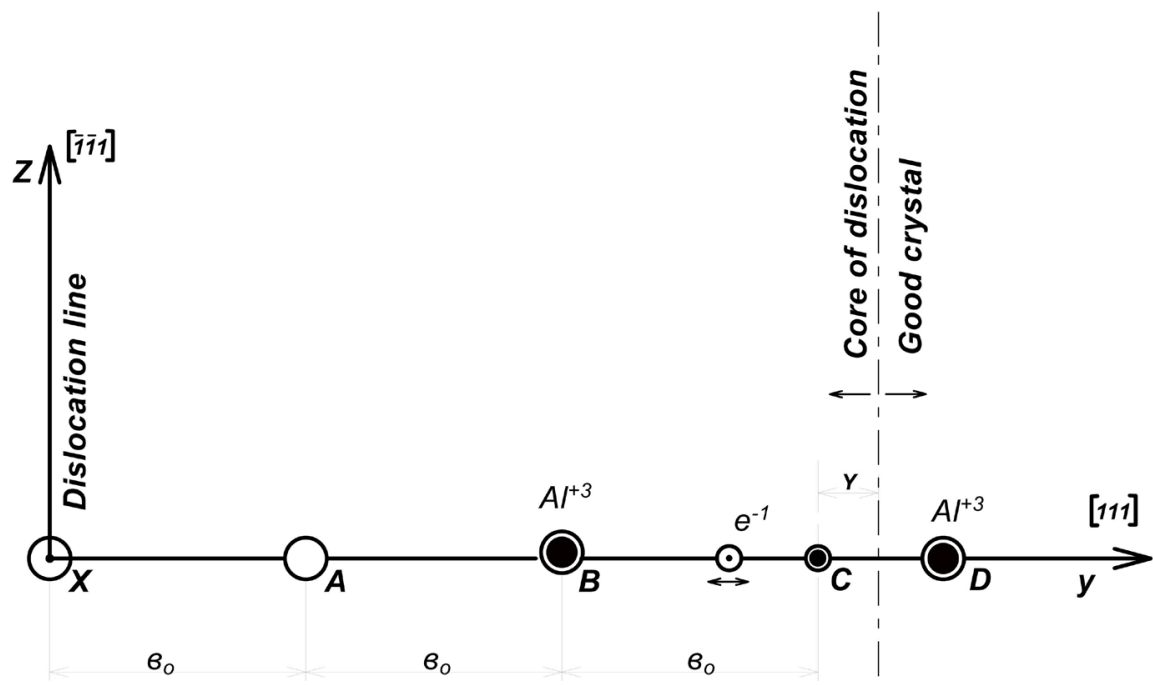

Figure 1. Linear structure in dislocation core in metals with face-centered lattice. $A, B, C$, $D$-positions of particles in octahedral interstitial sites. The axis $Z$ is along the direction

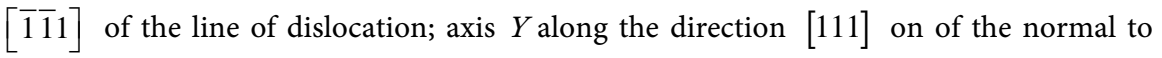
the dislocation line and the plane (111).

of the potential relief $V_{d}$ in the core of the defect does not allow to obtain from the distribution $N_{0}(\varepsilon)$ a dependence $V_{d}(\rho, \varphi, z)$ on the cylindrical coordinates with the help of [8].

A description of the dynamics of the above structure in the dislocation core and in a good crystal is possible using the theory of the LHOPS method ([2] [13], p.208) and [21] [22], in which cations and photoelectrons at positions $B$ and near $C$, with taking into account their orbital, spin and total moments in the corresponding interstices and nodes. We carry out a detailed description of this dynamics in a separate work.

\section{Discussion of the Results}

The mechanism of charge separation and the formation of linear structures in the generation of the dislocation nucleus are more realistic than the known adiabatic elastoplastic models of the continual theory of individual dislocations, atomic models of dislocation nuclei as it includes the natural processes of bremsstrahlung, photoelectric effect, and Coulomb interaction. In the framework of this model, we do not cut and do not move the material on both sides of the cut in the appropriate way, we do not remove or relax it after these operations, but we produce a natural weak shock on the free surface. Here we can consider standing electromagnetic waves not only between impurity ions, but between impurity ions and the free surface, intergranular, interphase boundaries, and also boundaries of deformation origin. In addition, the bremsstrahlung of photoelectron chains leads us to the plasma frequency of oscillations, the theory of solid-state plasma ([19], p. 539).

The analysis in Figure 1 also shows that the boundary between the dislocation 
core and the good crystal passes in the interval $C D$ and is separated from the dislocation line on which the centers of impurity ions lie at a distance $r_{\text {core }}=2 b_{0}+Y$, where $Y \leq C D-r_{\text {ion }}\left(\mathrm{Al}^{3+}\right)$ has yet to be found. It is also of interest to supplement the well-known statement for disordered media on the statistical homogeneity on the average [23] with statistical isotropy in the mean. In this case, the intermittent field must be periodic in time and create linear structures in two opposite directions.

We compare the experimental dependence of the EME pulse amplitude $\varphi_{\text {EME }}(\varepsilon)$ [8] with the current-voltage characteristic in the Frank-Hertz experiment ([12], p. 542), where the accelerated cathode electrons undergo inelastic scattering by $\mathrm{Hg}$ atoms when the anode potential of the tube coincides with the critical potential $\mathrm{Hg}$, and elastic scattering in the absence of this coincidence, i.e. the energy of the atoms varies discretely. In our case, with plastic deformation $\dot{\sigma}=$ const , the amplitude $\varphi_{\mathrm{EME}}(\varepsilon)$, and together with it the energy accumulated in the crystal, also varies discretely. The natural question arises: "How do the above described linear structures distributed between two impurity ions contribute to the accumulation of energy in the crystal?" The answer follows from the above: only thanks to the energy level scheme of the cation of the matrix peculiar to him alone. Let us hypothesize 2: The latent energy of a crystal accumulated in dislocation cores contains a component that is of an electromagnetic nature.

This raises a natural question: What is the relationship between bremsstrahlung intensity $I_{d}$ and the recoil momentum of the impurity ion $\boldsymbol{q}_{i i}$ and cation matrix $\boldsymbol{q}_{m i}$ on impact? Here we will use work [24] and consider in the generalized space of rectangular pulses the effect of a rectangular pulse of the displacements $L_{\Omega} u$ on the system "impurity ion-distribution of conduction electrons" at a certain point $\boldsymbol{r}$ in the traveling wave regime, where $L_{\Omega}=U_{-}(x)-U_{-}(x+\tau)$ is a linear operator of rectangular pulses; $U_{-}$ is jump operator. At the first stage $L_{\Omega} u$, at the time $t$, a dipole moment $d_{0}=e[u] \delta(t)$ occurs, where $[u]$ is a jump in the displacement of the impurity ion relative to the surrounding cations of the matrix, then the halo of conduction electrons around the impurity ion is redistributed over relaxation time $\tau_{r e} \approx(0.01 \div 0.03) b_{0} / v_{F} \approx 10^{-14} \div 10^{-15} \mathrm{~s}, \quad v_{F}=10^{6} \mathrm{~m} / \mathrm{s} \quad\left(b_{0}=0.7 a_{0}\right)$ before inverting $d_{0}=e[u] \delta\left(t+\tau_{r e}\right)$ to zero. Such relaxation at $\tau_{r e} \ll T_{\text {rep }}$ is a source of bremsstrahlung of an intermittent field, where the bremsstrahlung intensity is $I_{\gamma} \sim \square \exp \left(-t / \tau_{r e}\right) \sim \omega_{r e}^{4}$, where $\square=\Delta-\frac{1}{c^{2}} \frac{\partial^{2}}{\partial t^{2}} ; \omega_{r e}=\tau_{r e}^{-1}$ is the relaxation frequency of the conduction electron subsystem. At the second stage $L_{\Omega} u$, with $t+\tau$, the radiation algorithm is repeated, but this field changes sign. Here $\Delta z_{\mathrm{Mg}-\mathrm{Al}}=u_{\mathrm{Mg}}-u_{\mathrm{Al}} \sim\left|\boldsymbol{q}_{i i}-\boldsymbol{q}_{m i}\right|$. Hence $I_{\gamma} \sim\left(E_{0}^{i f}\right)^{2} \approx I_{d} \sim\left|\boldsymbol{q}_{i i}-\boldsymbol{q}_{m i}\right|^{2}$. In addition the vectors $\boldsymbol{q}_{i i}$ and $\boldsymbol{q}_{m i}$ are collinear and have the same direction, and the acceleration vectors of conduction electrons $\boldsymbol{a}_{e}$ and $\boldsymbol{q}_{i i}-\boldsymbol{q}_{m i}$ are also collinear, but directed towards each other, while it is well known from classical electrody- 
namics that $I_{d} \sim\left|\boldsymbol{a}_{e}\right|^{3}$, where $\left|\boldsymbol{a}_{e}\right| \approx v_{F} / \tau_{r e}$.

The process of charge separation and the formation of linear structures in the nuclei of linear defects can be described using a system of dynamic equations for the external currents of these charges according to [24] [25]. Here, for the collision of solids in the generalized space of rectangular impulses of shock loads, a discrete model of defect nuclei in crystalline materials is presented, where an undeformed perfect crystal is taken as Hilbert space of wave functions of the Schrödinger equation, and the core of a dislocation is the rigged Hilbert space of step functions as a combination of these functions of different sign, separated by a time interval. It was shown in [24] that a system of pairs of cations and photoelectrons knocked out of these cations appears in the core of a linear defect, and an intermittent field $E_{0}^{i f}$ in the form of a periodic sequence $E_{0}^{i f} \delta(t)$ and $-E_{0}^{i f} \delta(t+\tau)$ with the pulse repetition frequency $\omega_{\text {rep }}$ in the standing wave regime can interact through the chains of photoelectrons with the electron subsystem of the solid-state plasma. The features of the motion of charged particles and the interaction of chains of photoelectrons with the material's own plasma are taken out in a separate work.

\section{Conflicts of Interest}

The author declares no conflicts of interest regarding the publication of this paper.

\section{References}

[1] Cottrell, A.H. (1953) Dislocations and Plastic Flow in Crystals. 2nd Edition, Oxford University Press, Oxford.

[2] Van Buren, H.G. (1962) Defekty v kristallakh (Defects in Crystals). Izd. Inostr. Lit., Moscow.

[3] Kelly, A. and Groves, G.W. (1970) Crystallography and Crystal Defects. Longman, London.

[4] Reims, S. (1972) Theory of Many-Electron Systems. North-Holland Publishing Company, Amsterdam.

[5] Brown, R.A. (1966) Electron Distribution about an Edge Dislocation in a Metal. Physical Review, 141, 568. https://doi.org/10.1103/PhysRev.141.568

[6] Oqurtani, T.O. and Huggins, R.A. (1967) Theory of Electric Field Gradient Due to Conduction Electron Charge Density Redistribution around Screw Dislocation in Metals. Physica Status Solidi (b), 24, 301-311.

https://doi.org/10.1002/pssb.19670240130

[7] Friedel, J. (1952) XIV. The Distribution of Electrons Round Impurities in Monovalent Metals. The London, Edinburgh, and Dublin Philosophical Magazine and Journal of Science, 43, 153-189. https://doi.org/10.1080/14786440208561086

[8] Anokhin, A.O., Halperin, M.L., Gornostyrov, U.N., Katznelson, M.I. and Trefilov, A.V. (1994) On the Possibility of Localization of Electrons on Dislocations, Disclinations and at Grain Boundaries. Journal of Experimental and Theoretical Physics Letters, 59, 344.

[9] Shuvalov, L.A., Urusovskaya, A.A., Zheludev, I.S., Zalessky, A.V., Semiletov, S.A., 
Grechushnikov, B.N., Chistyakov, I.G. and Pikin, S.A. (1981) Modern Crystallography 4: Physical Properties of Crystals (V.4). Nauka, Moscow.

[10] Landauer, R. (1951) Conductivity of Cold-Worked Metals. Physical Review, 82, 520. https://doi.org/10.1103/PhysRev.82.520

[11] Orlov, A.N. (1983) Introduction to the Theory of Defects in Crystals. High School Press, Moscow.

[12] Kuzmichov, B.E. (1989) Laws and Formulas of Physics. Directory. Naukova Dumka, Kyiv.

[13] Stoneham, A.M. (1975) Theory of Defects in Solids. Electronic Structure of Defects in Insulators and Semiconductors. Claredon Press, Oxford.

[14] Akhiezer, A.I. and Berestetsky, V.B. (1969) Quantum Electrodynamics. Nauka, Moscow.

[15] Shibkov, A.A., Titov, S.A., Zheltov, M.A., Hasanov, M.F., Zolotov, A.E., Proskuryakov, K.A. and Zhigachev, A.O. (2016) Electromagnetic Emission during the Development of Macroscopically Unstable Plastic Deformation of a Metal. Physics of the Solid State, 58, 1-8. https://doi.org/10.1134/S1063783416010297

[16] James, H.M. and Lark-Horovitz, K. (1951) Localized Electronic States in Bombarded Semiconductors. Zeitschrift für Physikalische Chemie, 198, 107-126.

[17] Vainshtein, B.K., Fridkin, V.M. and Indenbom, V.L. (1979) Modern Crystallography Vol. 2: Structure of Crystals. Nauka, Moscow.

[18] Chernov, A.A., Givargizov, E.I., Bagdasarov, K.S., Kuznetsov, V.A., Demyanets, L.N. and Lobachev, A.N. (1980) Modern Crystallography (in 4 Volumes) V.3. The Formation of Crystals. Science, Moscow.

[19] Prokhorov, A.M. (1984) Physical Encyclopedic Dictionary. The Soviet Encyclopedia, Moscow.

[20] Honeycombe, R.W.K. (1968) The Plastic Deformation of Metals. Edward Arnold Ltd., Cambridge.

[21] Longuet-Higgins, H.C., Öpik, V., Pryce, M.H.L. and Suck, R.A. (1958) Studies of the Jahn-Teller Effect. II the Dynamical Problem. Proceedings of the Royal Society $A, 244,1-16$.

[22] Sloncewski, J.C. (1963) Theory of the Dynamical Jahn-Teller Effect. Physical Review, 131, 1596. https://doi.org/10.1103/PhysRev.131.1596

[23] Lifshitz, I.M., Gredeskul, S.A. and Pastur, L.A. (1982) Introduction to the Theory of Disordered Media. Nauka, Moscow.

[24] Busov, V.L. (2019) Dynamic Evolution Equations for the Cores of Linear Defects of Crystalline Materials in Colliding Solids. Physical Mesomechanics, 22, 91-96.

[25] Lifshits, E.M. and Pitaevsky, L.P. (1979) Theoretical Physics. V10. Physical Kinetics. Nauka, Moscow. 